\title{
La revisión en escritura colaborativa a través de un wiki: un estudio de caso ${ }^{1}$
}

\author{
Emmy Antonella González Lillo \\ Universitat Pompeu Fabra \\ Barcelona, España
}

\section{Resumen}

Debido al interés que reviste la enseñanza de la escritura, este estudio tiene como objetivo describir el subproceso de revisión que realiza un equipo de estudiantes chilenos en la escritura colaborativa de un ensayo en un wiki. Para realizar esta investigación de carácter exploratorio se utilizó un cuestionario de tipo mixto, se hizo un análisis de contenido del historial del proceso de escritura realizado en el wiki y se entrevistó a los participantes. Los resultados indican que los alumnos reconocen que la revisión debe contemplar principalmente la presencia de coherencia y cohesión. No obstante, no hay un proceso recursivo de escritura en el que vuelvan a subprocesos previos. Por esta razón, se requeriría de pautas que inciten a evaluar en la revisión, generando de este modo autonomía y autorregulación en el proceso de aprendizaje. Palabras clave: proceso de escritura; revisión; escritura colaborativa; wiki; Web 2.0.

\begin{abstract}
Revision in collaborative writing through a wiki: A case study

Due to the interest in the teaching of writing, this study aims to describe the revision sub-process that a group of Chilean students carry out in the collaborative writing of an essay in a wiki. Data was collected through the administration of a mix-item questionnaire, content analysis of the wiki writing process history, and interviews with the participants in the study. The results indicate that the students recognize that the revision sub-process should focus on coherence and cohesion of the text being revised. Nevertheless, there is no recursive writing process in which the students return to previous sub-processes. For this reason, guidelines are needed to train
\end{abstract}

\footnotetext{
${ }^{1}$ Este artículo se enmarca en el proyecto I+D+i ECODAL: Evaluación de la competencia discursiva de aprendices adultos plurilingües: detección de necesidades formativas y pautas para un aprendizaje autónomo (referencia: EDU2016-75874-P), dirigido por Carmen López Ferrero en la Universitat Pompeu Fabra (Barcelona). El proyecto ECODAL es financiado por el Plan Nacional de Investigación Científica, Desarrollo e Innovación Tecnológica 2016 del Ministerio de Economía, Industria y Competitividad español, la Agencia Estatal de Investigación (AEI) y el Fondo Europeo de Desarrollo Regional (FEDER).
} 
students to assess while revising, promoting autonomy and self-regulation in their learning process.

Key words: writing process; revision; collaborative writing; wiki; Web 2.0.

\section{Résumé}

Révision en écriture collaborative à travers un wiki : une étude de cas

En raison de l'intérêt que suscite l'enseignement de l'écriture, cette étude vise à décrire le sous-processus de révision qu'une équipe d'étudiants chiliens effectue dans la rédaction collaborative d'un essai dans un wiki. Pour mener à bien cette recherche exploratoire, un questionnaire de type mixte a été utilisé, une analyse de contenu de l'historique du processus d'écriture sur wiki a été réalisée et les participants ont été interrogés. Les résultats indiquent que les étudiants reconnaissent que l'examen devrait principalement considérer la présence de cohérence et de cohésion. Cependant, il n'y a pas de processus d'écriture récursif dans lequel ils reviennent à des sousprocessus antérieurs. C'est pourquoi des lignes directrices seraient nécessaires pour encourager l'évaluation dans le cadre de la révision, générant ainsi une autonomie et une autorégulation dans le processus d'apprentissage.

Mots-clés : processus d'écriture ; révision ; écriture collaborative ; wiki ; Web 2.0. 


\section{CóMO CITAR ESTE ARTÍCULO}

González, E. (2020). La revisión en escritura colaborativa a través de un wiki: un estudio de caso. Lenguaje, 48(2), 328-353. doi: 10.25100/lenguaje.v48i2.8804 


\section{INTRODUCCIÓN}

Escribir es una de las habilidades más necesarias que desarrollar en la vida de todo sujeto, a la vez que uno de los fenómenos más complejos de la actividad humana. Por lo tanto, la escuela ha de tener el propósito de formar niños y jóvenes competentes en producción escrita. A partir de esta premisa, aunque existen investigaciones sobre qué es la escritura y cómo debe ser enseñada aún quedan aristas por estudiar, como, por ejemplo, de qué modo el uso de las TIC puede mejorar su desarrollo. En efecto, la escritura digital colaborativa es un área de investigación incipiente, por lo que este trabajo pretende contribuir a ella presentando un estudio de caso que describe el subproceso de revisión en la escritura colaborativa a través de un wiki, así como también explora los valores que esta herramienta digital nos ofrece: colaborar y compartir en la construcción de conocimiento.

En este marco, es fundamental señalar en primer lugar que, puesto que la revisión es un subproceso de análisis de las estructuras formales y del contenido organizacional del texto, podría conformar una manera de enseñar por parte del profesor, pero también un método de aprendizaje del alumno. Al respecto, Álvarez (2010) comenta que el docente debería ser un agente que orienta y proporciona el andamiaje de las microdestrezas de escritura, otorgando poco a poco autonomía al escritor. Por consiguiente, se desprende que el foco pedagógico tiene que estar situado en la revisión como una función mental y discursiva que estimula al aprendiz a releer sus borradores y volver a trabajar sobre su pensamiento.

En segundo lugar, si bien en la actualidad se dispone de diferentes tecnologías y avances informáticos que proponen nuevos principios, también es cierto que se responde parcialmente a ellos. En este preciso sentido sería productivo estudiar cómo se podrían explotar los beneficios del aprendizaje colaborativo que han sido destacados por varios autores (Collazos y Mendoza, 2006; Guitert y Pérez-Mateo, 2013; Johnson, Johnson y Smith, 1991; Roselli, 2011; entre otros) y los valores que la Web 2.0 ofrece: colaborar en la construcción de conocimiento y compartirlo (O'Reilly, 2007; Peachey, 2009).

En los estudios del área es posible encontrar diversas propuestas que sugieren la utilización, por ejemplo, de blogs como recurso que favorece el aprendizaje a través de la escritura o que potencia el trabajo colaborativo mediante la escritura digital. En este trabajo se opta por la utilización de la herramienta wiki, puesto que pareciera ser una alternativa de la cual se puede obtener numerosos beneficios, no solo debido a que potencia el carácter multimodal de la comunicación, sino además porque sienta sus bases en la inteligencia colectiva y la idea de que el producto de un trabajo colaborativo será, generalmente, mejor que el de uno individual. Asimismo, explicita el proceso y subprocesos de escritura multimodal llevados a cabo por los escritores y permite analizar las revisiones que hace cada participante.

En consideración a lo expuesto, proponemos en este estudio abordar el subproceso de revisión en la escritura colaborativa a partir de una propuesta didáctica que usa un wiki de estudiantes chilenos de último año de educación secundaria. Para 
ello, nos hemos propuesto (a) indagar en las percepciones de los participantes en el estudio acerca del proceso de escritura colaborativa, en su participación en redes sociales y en su uso de sitios Web 2.0 y (b) caracterizar el proceso de revisión en la construcción de un ensayo colaborativo, a través del historial de wiki y del contraste entre las valoraciones de los estudiantes y de la profesora participantes en el estudio.

\section{MARCO TEÓRICO}

Para lograr los objetivos señalados en el apartado anterior, se presenta a continuación una síntesis del marco teórico al cual se ciñe este estudio, en el que se contemplan los estudios más relevantes en cuanto al proceso de escritura y subproceso de revisión; cuáles son las características y beneficios de la escritura colaborativa como práctica pedagógica; los principios de las herramientas Web 2.0 y definición de un wiki; y el desarrollo de la Web 2.0 en el contexto chileno.

\section{Proceso de escritura: la revisión}

La escritura es un fenómeno cultural que ha despertado el interés de muchos y, en consecuencia, ha sido una de las habilidades lingüísticas que más cambios ha evidenciado en su estudio: desde los enfoques gramaticales hasta los enfoques por proceso. Después de varias décadas de investigación acerca de esta actividad, hay un cambio de paradigma con Flower y Hayes (1981), quienes intentan descubrir qué ocurre en la mente del escritor. Posteriormente, Scardamalia y Bereiter (1992) plantearon los modelos de "decir el conocimiento", propio de los escritores novatos y "transformar el conocimiento", característico de los escritores expertos. Actualmente, se recogen y admiten solo algunas ideas de estas primeras investigaciones, pero es innegable que a partir de ellas se inicia y sientan las bases de lo que ahora es una amplia tradición en el estudio del proceso de escritura.

En este sentido, a partir del enfoque cognitivo nacen otras tres perspectivas teóricas y metodológicas desde donde abordar la composición escrita: la sociocognitiva, la sociocultural y, más recientemente, la regulación socialmente compartida (Castelló, Bañales y Vega, 2010). De acuerdo con Arenas (2017), estos cuatro enfoques se distinguen teóricamente por la manera en que comprenden la regulación y las funciones que se le asignan a los factores metacognitivos, motivacionales y contextuales y metodológicamente varían en las situaciones que permiten recoger los datos. La autora indica que, así como la investigación de carácter cognitivo se caracterizó por comprender el proceso de composición como un problema cuya solución estaba estrechamente ligada a subprocesos de planeación, textualización y revisión, el enfoque sociocognitivo se caracteriza por añadir al análisis de la escritura la influencia de componentes sociales.

Por otro lado, el enfoque sociocultural integra procesos en los que se reconoce la importancia de la interacción con otros. Finalmente, el enfoque que estudia la producción escrita como un proceso de regulación socialmente compartida se ampara en el estudio de procesos de escritura colaborativa, analizando principalmente los 
papeles que asumen los escritores, así como las interacciones que se efectúan en la planificación, la textualización y la revisión del texto. En relación con esta última idea, Álvarez (2010) indica que el proceso de producción supondría revisar las estructuras formales y del contenido organizacional del texto no solo al final, sino también en la planificación y redacción debido a que solo entonces se remodelarían los esquemas mentales y se transformaría el conocimiento.

Ahora bien, en este trabajo nos centramos específicamente en el subproceso de revisión, el cual, siguiendo a Álvarez y González (2012), consistiría en evaluar y corregir, al mismo tiempo que en transformar el conocimiento y construir pensamiento. En este sentido, durante este subproceso el escritor debe propender a cumplir ciertas condiciones: la adecuación a la intención comunicativa; alcanzar coherencia y cohesión; y adaptarse al perfil del destinatario (considerando sus conocimientos del contenido y lingüísticos). La revisión es un saber hacer que interviene en todas las tareas: desde la planificación a la textualización y escrito final. En consecuencia, puesto que tiende a la mejora, desde el punto de vista pedagógico, corresponde a una tarea primordial. Sin embargo, en el contexto de aula lo anterior se cumple parcialmente. Esta deficiencia en la enseñanza-aprendizaje de la escritura queda demostrada en múltiples y diversos problemas: desde los que afectan a cuestiones formales y gramaticales, hasta los relacionados con la selección de la información relevante, la claridad y el orden en la exposición de las ideas.

\section{Escritura colaborativa}

La escritura colaborativa se ha de entender como la coautoría, es decir, dos o más personas producen un texto en conjunto, llevando a cabo todo el proceso de manera compartida (McDonough, De Vleeschauwer y Crawford, 2018; Storch, 2013, 2019a). Se caracteriza por ofrecer varias ventajas en comparación con la escritura individual, pues, aunque ambas modalidades comparten la adaptación a las estructuras formales del tipo de texto, la escritura colaborativa supone la coordinación y comunicación entre los participantes del equipo, esto es, la interdependencia positiva. Estas acciones implican que, además de la ejecución de los subprocesos regulares de la escritura, en el caso del trabajo colaborativo ${ }^{2}$ se agregue una tarea paralela: la discusión y el logro de acuerdos mientras se construye el texto.

Ribas (1997) ya señalaba que escribir en pequeños grupos favorecería tanto la explicitación de los contenidos sobre escritura, como también la toma de conciencia del proceso que se está llevando a cabo. En efecto, escribir colaborativamente propicia la regulación del aprendizaje. Panadero et al. (2013) señalan que se pueden distinguir

\footnotetext{
2 Para evitar confusiones, será conveniente precisar que algunos autores -Collazos y Mendoza, 2006; Fathman y Kessler (citados en Trujillo, 2002); Lucero, 2003; Zañartu, 2003; entre otros- hacen la distinción entre aprendizaje cooperativo y aprendizaje colaborativo. El primero es el trabajo en grupo, entendido como aquel en el que se estructura la clase de modo que todos los estudiantes interactúen e intercambien información, pero puedan a la vez ser evaluados de forma individual por su trabajo. En cambio, por otra parte, el aprendizaje colaborativo responde a un trabajo en equipo, lo que más bien apunta a cómo la interacción social conlleva beneficios para el aprendizaje.
} 
dos tipos de regulación: la corregulación, esto es, la coordinación de las dinámicas de grupo dirigida principalmente por una persona y la regulación socialmente compartida, es decir, negociación entre los participantes de un equipo donde se construyen relaciones de igualdad puesto que todos los miembros poseen objetivos comunes (Arenas, 2017). Es precisamente aquella regulación o toma de conciencia la primera evidencia palpable de los beneficios de la escritura colaborativa: mientras se escribe, se debe argumentar y retroalimentar inmediatamente, por lo que hay una constante revisión y monitoreo que permiten decidir qué contenidos incluir, cómo estructurar el texto y qué recursos pueden clarificar las ideas expuestas. A partir de esa discusión entre los participantes se dará lugar a un producto mejor escrito (Guzmán y Rojas-Drummond, 2012; Uribe, Ramírez y Henao, 2017). Otros atributos de la escritura colaborativa que indican Ubilla, Gómez y Sáez (2017) son tres: 1) afectivamente el estudiante y el grupo sienten más confianza en sí mismos y más motivación; 2) se crea un ambiente de trabajo distendido; y 3) existe una audiencia auténtica que permite a los aprendices conocer lo que los lectores comprenden de su texto.

Ahora bien, el desafío es mayor cuando integramos los entornos virtuales, que se caracterizan por ser adaptables, interactivos y de amplia utilidad, puesto que pueden ser usados dentro y fuera del aula. En efecto, Calle (2018) lo indica acertadamente al señalar que:

la escritura, hoy, significa mucho más que simplemente trabajar con texto alfabético o con las páginas impresas, las aplicaciones informáticas y espacios de publicación digital nos permiten tejer múltiples códigos y posibilidades para producir textos que pueden ser interactivos, hipertextuales y bastante potentes. (p. 336).

La red otorga a los estudiantes el acceso a herramientas que complementan tanto su escritura como su aprendizaje de forma atractiva, pues satisfacen sus intereses personales. Asimismo, el uso de recursos digitales exige el progreso de una reflexión y análisis de los contenidos a los que se accede.

A la luz de lo planteado, el aprendizaje colaborativo en entornos virtuales contemplará entre sus beneficios los que siguen: la mejora de habilidades de alto orden de pensamiento crítico; la autonomía en el aprendizaje y las colaboraciones se vuelven más efectivas; y una mejora sustancial de habilidades sociales personales y de grupo (Cabrera, 2004).

\section{Herramientas Web 2.0}

Es natural que conforme pasa el tiempo nuestra sociedad experimente cambios en todas sus esferas. Es así como en los inicios del siglo veintiuno notamos el vertiginoso empoderamiento de las nuevas tecnologías en el espacio social, económico, político, cultural y también, por supuesto, educativo. De ahí que se abriera paso a la, por entonces, nueva web informativa: la Web 2.0. Tal expresión fue creada por O'Reilly (2007), y el mismo autor señala que el éxito de la web tiene como objetivo aprovechar 
la inteligencia colectiva. Según Latorre (2018), aunque actualmente existe una web 3.0 e incluso una 4.0, "la web 2.0 permite compartir información. Y aquí estamos, de momento la mayor parte de los consumidores" (p. 2).

Es en dicho entorno donde la escritura colaborativa toma lugar. Como indica Storch (2019b), además de realizarse en clase frente a frente, cada vez más se hace de manera online, donde los estudiantes usan plataformas como blogs, foros virtuales, documentos virtuales, wikis, etc. para interactuar con otros y generar aprendizajes y conocimientos compartidos por quienes integran esa comunidad.

En esta línea de ideas, Cassany y López (2005) señalaban que el paso a entornos digitales no implica exclusivamente cambiar tipos textuales. En efecto, los hipertextos requieren otras habilidades: tener capacidad crítica para discriminar si la información en Internet es fiable respecto a la que no lo es; construir documentos hipertextuales que permitan crear distintos itinerarios para el lector; poder enlazar los diferentes componentes verbales con vínculos entre sí y con componentes no verbales; desarrollar habilidades de trabajo en equipo y de cooperación con coautores, porque en la red los discursos se interrelacionan con otros, formando un entramado global y único; escribir en contextos de comunicación reales, etcétera.

Ahora bien, Pujolà (2013) advierte que el desarrollo didáctico de los materiales digitales deberá tener en cuenta aspectos como la interactividad, es decir, la relación entre los alumnos con el material interactivo y la interacción entre profesor-alumnos y entre los propios alumnos. También, habrán de considerarse la opcionalidad, entendida como la posibilidad de que los alumnos puedan llegar al mismo objetivo por distintos caminos y el feedback, esto es, el vínculo entre el alumno, el proceso de aprendizaje y su resultado para promover un aprendizaje que ayude al aprendiz a ser más autónomo.

Una de las herramientas disponibles en los entornos digitales es el wiki: un tipo de página web que permite a los usuarios crear textos e hipertextos colaborativamente de forma rápida y fácil. Se caracteriza por posibilitar que varios usuarios editen un texto de manera asincrónica y provee un historial de los cambios realizados por medio del cual se puede acceder, incluso, a las primeras versiones del documento. Finalmente, obliga a los estudiantes a comprometerse con lograr la meta que tienen en común, promoviendo el desarrollo del concepto de coautoría. Duffy y Bruns (2006) señalaron que el wiki corresponde a un espacio de gestión del conocimiento, que se caracteriza por ser altamente flexible, al mismo tiempo que un medio muy efectivo de generar e intercambiar información por medio del esfuerzo colaborativo.

\section{La Web 2.0 en Chile}

Con respecto al uso de la Web 2.0 en Chile conviene comentar someramente que la brecha digital ha afectado el dominio de estos recursos $\mathrm{y}$, en consecuencia, la pertenencia de los alumnos al grupo de "residente o visitante digital" (White y Le Cornu, 2011). Sin embargo, actualmente, el país ha reducido de modo considerable la 
desigualdad que durante años aquejó a la ciudadanía ${ }^{3}$ y es así como en el contexto del Aprendizaje de Lenguas Asistido por Computador (CALL por su sigla en inglés), ha surgido una tendencia a desarrollar la escritura digital.

Aunque predominantemente existen estudios que abordan el uso del wiki en la educación superior, hay también otras propuestas que lo hacen en la enseñanza secundaria. A título de ejemplo, cabe destacar la secuencia didáctica de Tapia, Correa, Ortiz y Neira (2012), cuyo objetivo fue desarrollar las habilidades de producción colaborativa de los participantes a partir del enfoque de proceso de escritura, el aprendizaje colaborativo y la comunicación mediada por ordenador. A partir de esta propuesta, los autores indican que la utilización de un wiki permite a los estudiantes elaborar un texto en equipo, pero al mismo tiempo fomenta la utilización de otras herramientas. De modo similar, la propuesta de escritura colaborativa de un texto académico en inglés planteada por Ortiz y Ferreira (2014) promueve el uso de la tecnología en la enseñanza de una segunda lengua, en este caso, del inglés como lengua extranjera (EFL).

\section{Metodología}

Este estudio se presenta como una investigación de carácter exploratorio que sigue una metodología cualitativa (Cohen, Manion y Morrison, 2011; Dörnyei, 2007). Dada la naturaleza de los datos recogidos, hemos optado por realizar un estudio de caso (Creswell, 2007, 2012) que analiza el subproceso de revisión en la producción escrita de un wiki llevado a cabo por cuatro estudiantes. El contexto de aplicación se sitúa en un establecimiento particular subvencionado de Viña del Mar (Chile). Dicho centro imparte clases solo al sector secundario, contando con tres grupos por nivel educativo.

La actividad que se analiza en este estudio corresponde a una tarea de escritura evaluada en la asignatura de Lengua y Literatura. Este grupo ${ }^{4}$ está conformado por 32 estudiantes cuyas edades oscilan entre 17 y 18 años. Sin embargo, pese a que se recogieron las respuestas del cuestionario y los historiales del wiki de 27 jóvenes, por cuestiones de espacio hemos acotado como muestra de la investigación el trabajo realizado por el "Equipo 1".

Este equipo está conformado por cuatro estudiantes, un hombre y tres mujeres, todos de origen hispanohablante. Su selección obedece a los siguientes criterios: poseemos los consentimientos informados firmados por ellos y por sus tutores legales;

\footnotetext{
${ }^{3}$ Según los datos obtenidos en la Novena Encuesta de Accesos y Usos de Internet (Subsecretaría de Telecomunicaciones de Chile, 2017), un 87,4\% de la población tiene acceso a Internet, pero solo un $44 \%$ de los hogares dispone de conexión fija. Los resultados son alentadores en comparación con estudios anteriores, pero no son suficientes. El hecho de que el $66 \%$ de los hogares no tengan acceso a la red impide a aquellos categorizados como visitantes digitales traspasar la barrera de la residencia digital. De esta circunstancia recae, entonces, en la educación chilena el compromiso de facilitar a los niños y jóvenes más vulnerables del país el acceso a las TIC (Tecnologías de la información y la comunicación) y la correspondiente alfabetización digital.

${ }^{4}$ Hemos indicado en una nota al pie anterior que existe una diferencia entre equipo y grupo. Sin embargo, por cuestiones de sustitución léxica utilizaremos los términos indistintamente.
} 
asistieron a todas las clases de la secuencia didáctica; la profesora indicó en su diario docente que es el equipo que mejor trabajó; es uno de los grupos que presenta numerosas correcciones e intervenciones en el wiki; todos los integrantes participaron más de una vez; se involucraron durante todo el proceso y no solo después de la corrección del borrador.

La docente a cargo del curso ha trabajado con estos estudiantes durante cuatro meses y posee un conocimiento bajo acerca de las TIC, pues señaló utilizar regularmente solo su cuenta de correo electrónico y las herramientas básicas de Microsoft Office. Además, reveló no haber utilizado antes un wiki, por lo que hemos construido en conjunto la secuencia didáctica para la tarea de escritura que evaluó. Nuestra participación consistió, en primer lugar, en capacitar a la docente para la utilización de la plataforma PbWorks. Posteriormente, en conjunto, relevamos cuáles eran los Contenidos Mínimos Obligatorios (CMO) que la docente debía cubrir de acuerdo con el programa de educación para el nivel. Si bien es cierto que durante toda la enseñanza secundaria el desarrollo de la competencia escrita es un objetivo transversal, los géneros asociados a su desarrollo cambian de acuerdo con el nivel de estudio.

En concordancia con lo anterior, la profesora se encargó de diseñar los materiales asociados al cumplimiento de los objetivos de aprendizaje (CMO) y el instrumento de evaluación (pauta de cotejo), el que tuvo como indicadores de logro: 1) la adecuación de aspectos formales (realizar un texto coherente con el tema abordado; desarrollar un texto argumentativo con la estructura asignada; utilizar bibliografía diversa para apoyar la tesis; establecer preguntas e inquietudes que responden a la temática y utilizar de manera correcta normas ortográficas vigentes); 2) seguir el proceso de escritura (desarrollar el trabajo de manera colaborativa, todos los integrantes aportan ideas significativas al ensayo; escribir de manera paulatina en el tiempo, por lo que se presentan avances en las revisiones pactadas; presentar avances correspondientes a las fechas dadas en las instrucciones iniciales; realizar ingresos al wiki para aportar ideas): y 3) adecuación al género discursivo Ensayo argumentativo (elaborar una tesis en la cual se exponga de manera eficiente su punto de vista; elaborar argumentos que respalden a la tesis de manera firme y coherente; utilizar bibliografía especializada en el tema y realiza citas cuando se requiere; realizar una introducción y conclusión del tema rescatando los principales puntos del ensayo; y realizar el texto con la extensión acordada). En paralelo, nosotros preparamos un tutorial para los estudiantes para la utilización de la página web. Los tiempos para realizar la actividad fueron consensuados con la docente, tanto para que existiera una etapa de evaluación formadora, como una sumativa.

Para la realización de esta investigación hemos utilizado dos tipos de instrumentos de recogida de datos: principales y complementarios. En la primera categoría incluimos el historial del $w i k i^{5}$, un cuestionario mixto y dos entrevistas

\footnotetext{
${ }^{5}$ Se ha utilizado el sitio https://my.pbworks.com/, puesto que es gratuito y de fácil acceso.
} 
semiestructuradas, mientras que en la segunda consideramos pertinente la utilización de un diario de notas de la profesora ${ }^{6}$ y una grabación de audio de la clase en la que se proporcionan las instrucciones del proyecto de escritura a los estudiantes.

La aplicación del cuestionario en línea nos proporcionó el análisis de las necesidades de los estudiantes y, al mismo tiempo, la obtención de algunos datos de carácter general (nombre, edad, lugar de procedencia, etc.). La primera parte del cuestionario, de carácter cuantitativo, midió a través de escalas de Likert las percepciones de los estudiantes sobre la escritura y el trabajo colaborativo. Para analizar los resultados se utilizó estadística descriptiva. La segunda parte, de orden cualitativo, midió la actividad que los jóvenes tienen en la red y la técnica utilizada para su análisis fue el análisis de contenido.

El segundo paso fue aplicar la secuencia didáctica diseñada con la profesora. La primera clase consistió en la presentación de una síntesis sobre cuál es el proceso de escritura y sus subprocesos. Realizado esto, se hizo una inducción a la tarea de escritura que consistió en realizar un ensayo argumentativo. Se ha trabajado con este género discursivo para no interferir en la planificación anual de la asignatura. La tarea de escritura fue la siguiente: "Realizar un ensayo, según la estructura del género analizada en clases (presentación del tema, tesis, desarrollo, conclusión), con elección libre del tema. El escrito debe contener entre 1500 y 3000 palabras y se debe realizar en el wiki, cuya invitación recibirán vía correo electrónico".

Dadas las instrucciones se formaron ocho equipos de trabajo (compuestos por tres o cuatro estudiantes) y se les enviaron las invitaciones para la edición de la herramienta. Los equipos de trabajo tuvieron un plazo de cuatro semanas para llevar a cabo la tarea de escritura. Durante este tiempo se realizó un seguimiento del historial de cada uno de los wikis. La plataforma utilizada permite observar cuáles son los cambios ejecutados por cada uno de los estudiantes, con lo que se hizo un análisis de contenido.

En primer lugar, se etiquetó cada uno de los cambios realizados por cada uno de los participantes. Posteriormente, se generaron categorías preliminares que dieron cuenta del proceso llevado a cabo con las ideas que se elaboraron, mantuvieron, reformularon, sustituyeron o suprimieron. Finalmente, se establecieron categorías generales que agruparon las acciones llevadas a cabo por los estudiantes tanto durante el proceso de escritura en general (acceso al conocimiento, planificación, producción, revisión y comentarios de carácter social), como del subproceso de revisión en particular (revisiones a nivel de contenido, párrafo, oración, palabra, sílabas o letras o paratextos).

Las entrevistas aplicadas tanto a estudiantes como a la profesora participantes se estructuraron en función de tres ejes: el proceso de escritura y la revisión, la escritura colaborativa y el dominio de la herramienta Web 2.0 wiki y para su análisis la técnica

\footnotetext{
${ }^{6}$ Se solicitó a la profesora llevar un diario docente en el cual registró las actividades realizadas durante el periodo de investigación, los tiempos asignados, los estudiantes asistentes y observaciones importantes, como, por ejemplo, según la percepción de la profesora, qué equipos mostraron más compromiso a lo largo del proyecto de escritura.
} 
utilizada también fue el análisis de contenido. Finalmente, debido a que el estudio involucró a personas, y que estas eran menores de edad, hemos tenido en consideración ciertas cuestiones éticas siguiendo el Code of Ethics de la American Educational Research Association (AERA Council, 2011) para asegurar el respeto y resguardo de los participantes.

\section{ANÁlisis DE LOS RESUltados}

En el presente apartado se expondrán los datos obtenidos a través de los instrumentos utilizados y se valorará los resultados a la luz de los objetivos propuestos inicialmente y del marco teórico expuesto.

\section{Percepciones de los estudiantes acerca del proceso de escritura colaborativa y su participación en sitios Web 2.0}

El cuestionario aplicado da cuenta de las percepciones sobre el proceso de producción escrita y el trabajo colaborativo que poseen los alumnos. Los resultados coinciden con estudios previos acerca de las actitudes negativas o rechazo que presenta el estudiantado frente a las tareas de escritura (Caldera y Escalante, 2006; De Caso y García, 2006): tres estudiantes indicaron que no les gusta escribir y tan solo una participante señaló que sí le agrada. Estos datos son fundamentales para comprender el rendimiento del equipo, ya que, tal como indica el Grupo Didactext en su modelo de escritura (2003), existe una dimensión que responde a un componente motivacional. Es, pues, la motivación del escritor la que inicia, rige, mantiene o detiene su desempeño. Ciertamente, hay una relación de dependencia entre dicho desempeño y la actitud del estudiante, por lo que el profesor también adquirirá un papel sustancial como agente que incite a una predisposición positiva.

Con relación al proceso y subprocesos de escritura, la mayoría de los participantes indica que tienden a buscar información acerca del tema, pero solo dos estudiantes planifican, mientras que los otros dos escriben libremente, de modo similar a una corriente de la conciencia. Estos resultados demuestran que no han internalizado completamente todos los subprocesos de escritura, pese a que, como se ha indicado previamente, durante toda la enseñanza secundaria ha sido una habilidad que se habría intentado desarrollar en ellos, según los CMO de las Bases Curriculares (Ministerio de Educación, 2015).

En relación con la revisión de forma, la tendencia es preocuparse por la ausencia de faltas ortográficas, pero no existe el mismo cuidado por la distribución de la información, ya que los cuatro participantes tienden a no revisarla. Tampoco hay una preocupación por la utilización adecuada de conectores; dos de los alumnos manifiestan que nunca verifican el uso correcto o apropiado. Estaríamos, pues frente a escritores inexpertos que creen que la ortografía y la gramática son algunos de los aspectos más relevantes en el proceso de escritura. A su vez, este descuido por el contenido da cuenta de que, una vez realizada la planificación, los escritores no 
vuelven atrás, ni reestructurarían el texto, sino que mantienen el esquema que han diseñado de principio a fin. Este dato es de suma relevancia, puesto que se colige que las revisiones de los estudiantes no consideran la relectura de sus textos y, por lo tanto, tampoco la reescritura.

Por último, ninguno de los estudiantes suele recurrir a otra persona para que revise o lea lo que han escrito, ya sea un adulto o un profesor. Solo dos jóvenes muestran tendencia a pedir ayuda a compañeros. Tomando como base este dato, podemos afirmar que los estudiantes no se preocupan por que sus textos sean comprensibles para otros, quedándose en la "prosa del escritor"7 (Flower, 1979). En este sentido, cabe observar y controvertir la idea de que este tipo de escritura es incorrecta en sí misma. El hecho de ser más libre y altamente dependiente del contexto para su comprensión no la hace mejor o peor que la prosa del lector. No obstante, su uso en la redacción de trabajos académicos es inadecuado en el entendido de que debieran predominar las funciones epistémica y comunicativa de la escritura.

De cara a los soportes y recursos que utilizan en el proceso de escritura, las respuestas arrojan que los cuatro estudiantes usan con frecuencia procesadores de textos, sin embargo, otras herramientas digitales no son utilizadas en medida similar. Dos participantes recurren siempre a diccionarios como el Diccionario de la Lengua Española o WordReference, pero los dos restantes los emplean ocasionalmente. Las imágenes, en cambio, son utilizadas por todos los alumnos en mayor o menor medida. No obstante, los vídeos $\mathrm{y}$, sobre todo, los archivos de audio son parcialmente utilizados.

Finalmente, respecto al uso y la participación en la Web 2.0, todos los estudiantes tienen una cuenta en una o más redes sociales como Facebook, Instagram o Twitter, pero no utilizan otros sitios en donde puedan compartir información. Solo uno de los participantes informa estar registrado en un sitio de blog. Estos resultados no sorprenden si los analizamos a la luz de estudios previos que indican también el alto uso de los espacios sociales digitales. Así lo demostraron García, López y García (2014) cuando expusieron que las redes sociales eran los sitios que más frecuentaban los adolescentes españoles, especialmente aquellos que se situaban entre los 15 y 17 años y que tal implicación no presentaba diferencias significativas según el tipo de educación ni la clase económica a la que pertenecían. Por su lado, el contexto chileno no difiere en gran medida de esa situación. A partir de la última encuesta realizada por la Subsecretaría de Telecomunicaciones de Chile (2017), se podría inferir que las redes sociales también son algunos de los sitios que más frecuentan los jóvenes, pues más del $50 \%$ de la población entre 16 y 29 años reconoció usarlos. De estas respuestas se comprende que ninguno de los alumnos haya usado antes un wiki.

\footnotetext{
${ }^{7}$ Se distingue "prosa del escritor" de "prosa del lector", en tanto la primera representa el pensamiento del escritor con significados personales y la segunda expresa por escrito un mensaje dirigido a un lector que no necesariamente debe recurrir al contexto para comprender el texto.
} 


\section{Caracterización del subproceso de revisión en el historial del wiki}

La tarea de escritura, cuyo producto final fue un ensayo realizado a través de un wiki, permitió observar que la mayoría de los aportes realizados por los estudiantes en el proceso de escritura se registran primordialmente en la segunda fase de producción, pues, aunque varios equipos presentan intervenciones en la primera, muchas de estas corresponden a comentarios de tipo social y exploración de la herramienta. Por lo tanto, los jóvenes no han realizado un proceso de producción paulatino, sino que una vez que la profesora controló el avance del borrador luego de la fase 1, comenzaron a desarrollar los textos con mayor responsabilidad y frecuencia (ver Tabla 1).

Tabla 1. Seguimiento de los comentarios y versiones por equipos de trabajo

\begin{tabular}{|l|l|l|l|l|l|}
\hline \multirow{2}{*}{ Equipo } & \multicolumn{2}{|l|}{${\text { Fase } 1^{8}}^{-}$} & \multicolumn{2}{|l|}{ Fase $2^{9}$} & Total \\
\cline { 2 - 6 } & $\begin{array}{l}\text { Comentarios } \\
\text { sociales }\end{array}$ & $\begin{array}{l}\text { Versiones del } \\
\text { texto }\end{array}$ & $\begin{array}{l}\text { Comentarios } \\
\text { sociales }\end{array}$ & $\begin{array}{l}\text { Versiones del } \\
\text { texto }\end{array}$ & \\
\hline $\mathbf{1}$ & $\mathbf{1}$ & $\mathbf{8}$ & $\mathbf{2}$ & $\mathbf{3 5}$ & $\mathbf{4 6}$ \\
\hline 2 & 5 & 2 & 12 & 30 & 49 \\
\hline 3 & 0 & 8 & 0 & 8 & 16 \\
\hline 4 & 5 & 4 & 7 & 23 & 39 \\
\hline 5 & 3 & 0 & 0 & 17 & 20 \\
\hline 6 & 0 & 0 & 0 & 17 & 17 \\
\hline 7 & 3 & 6 & 1 & 20 & 30 \\
\hline 8 & 2 & 0 & 0 & 7 & 9 \\
\hline
\end{tabular}

Fuente: elaboración propia

Respecto a los datos ilustrados cabe indicar de modo general que se advierte que los estudiantes están acostumbrados a escribir con la única finalidad de ser evaluados, pues, aunque se les propone que la elección del tema es libre, lo que supondría mayor motivación, no es hasta el control de avances de la profesora que los jóvenes toman un papel más activo en la construcción del texto. Esto indicaría que la motivación es extrínseca al tópico y está condicionada por la futura calificación que obtendrán. Es decir, prevalece una fuerte cultura de la heteroevaluación y la evaluación sumativa ${ }^{10}$; hay poco autocontrol y desarrollo de la auto- y corregulación. La evaluación sería vista por los alumnos como un medio intimidatorio que los obligaría a producir. Efectivamente, solo tres grupos presentan avances más significativos en la fase 1, entre ellos, el equipo de trabajo 1 (destacado en negrita en la Tabla 1), cuyo wiki es el objeto de análisis de este trabajo.

\footnotetext{
${ }^{8}$ Fase 1: periodo comprendido entre la invitación al wiki y la entrega del primer borrador para la revisión de la profesora.

${ }^{9}$ Fase 2: periodo comprendido entre la retroalimentación de la profesora y la edición final del wiki.

${ }^{10}$ Entendemos por heteroevaluación la valoración realizada por el docente hacia los estudiantes y por evaluación sumativa la valoración realizada al final de un ciclo de aprendizaje (López, Martín, Esteve y Atienza, 2019).
} 
De parte de la profesora es interesante observar que hay una evaluación formadora ${ }^{11}$ que responde más a una constatación de avances y que solo queda de manifiesto una vez. Si bien ella indica en su diario docente que realiza retroalimentaciones de manera oral a los jóvenes, vemos en ello un inconveniente: en la mayoría de los grupos faltó uno o más integrantes a esta instancia, por lo que no tuvieron acceso directo a ese feedback o bien, debido a que la oralidad es evanescente, muchas de las sugerencias que la maestra hizo pudieron ser obviadas $u$ olvidadas. Consideramos que es primordial dejar un registro de la evaluación formadora, ya que permitirá cambiar la concepción del estudiante frente a este concepto y comenzar a verlo como un proceso y no como un acontecimiento al término de la tarea.

Para tener un análisis más detallado de los procesos realizados por los estudiantes, hemos seleccionado, tal como se indicó previamente, un equipo de trabajo ("Equipo 1"), cuyo historial refleja que la mayoría de los aportes fueron hechos por solo una integrante del grupo. Las demás intervenciones se reparten entre los tres participantes restantes de forma desigual (Figura 1).

Fuente: elaboración propia

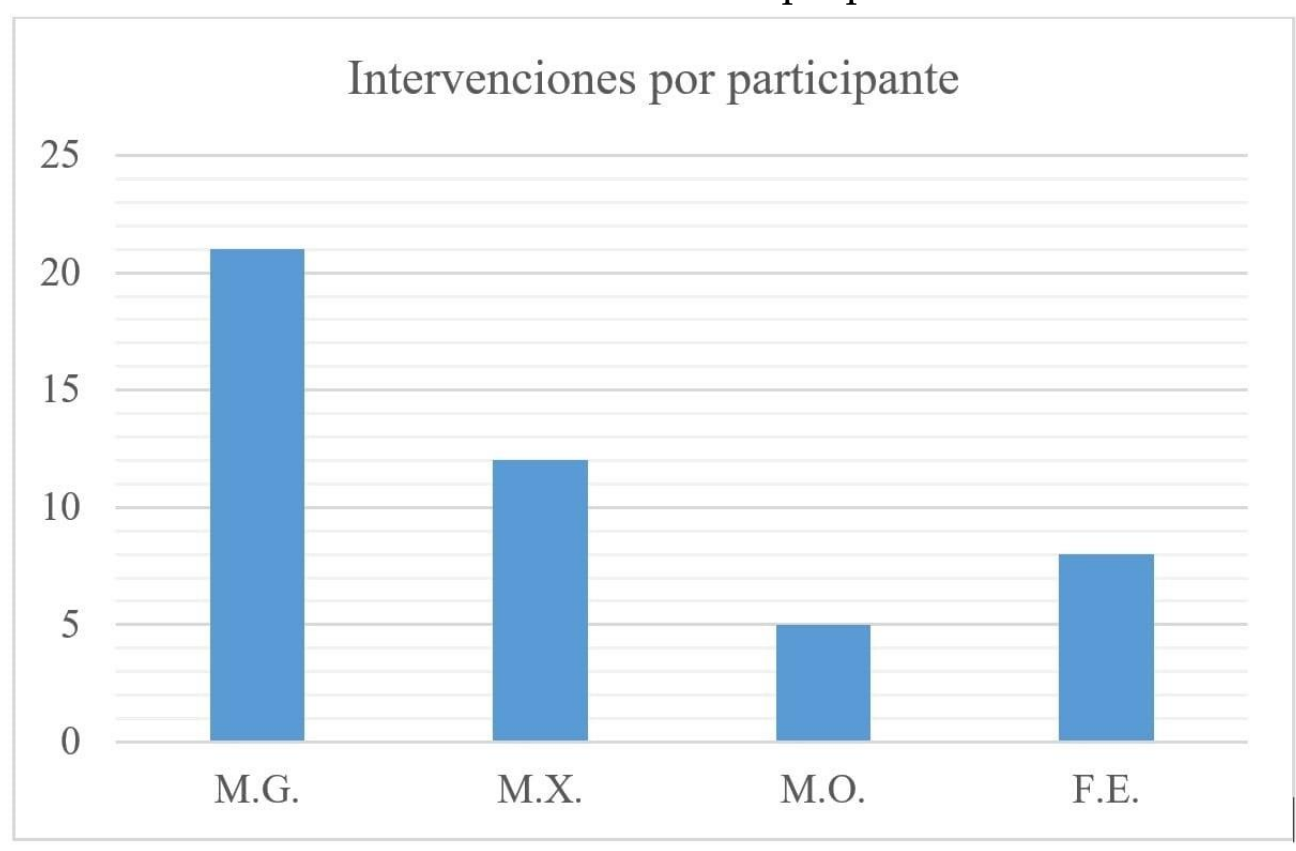

Figura 1. Intervenciones en el wiki por participante del Equipo 1

A partir de este recuento quisimos explorar si el número de intervenciones de cada participante tiene relación con el grado de implicación en el trabajo, pues al analizar los cambios que cada participante registra advertimos que una intervención puede tener más de una finalidad. Por ejemplo, en una intervención en el wiki, la participante M.G. realizó más de una acción: planificó, proporcionó información y

\footnotetext{
${ }^{11}$ Entendemos por evaluación formadora un enfoque, cuya atención se centra en la evaluación como proceso para orientar la enseñanza-aprendizaje y favorecer la toma de decisiones oportunas en beneficio de los estudiantes (López et al., 2019).
} 
corrigió errores ortográficos. En la Tabla 2 resumimos los aportes de cada integrante según el análisis de contenido hecho en cuanto al subproceso de escritura ejecutado.

Tabla 2. Intervenciones de los participantes del Equipo 1 según subprocesos de escritura realizados

\begin{tabular}{|l|l|l|l|l|l|}
\hline \multirow{2}{*}{ Intervenciones } & \multicolumn{4}{l}{ Participante } & \multicolumn{4}{l|}{} \\
\cline { 2 - 7 } & M.G. & M.X. & M.O. & F.E. & Total \\
\hline Acceso al conocimiento & 2 & 0 & 0 & 1 & 3 \\
\hline Planificación & 5 & 1 & 0 & 2 & 8 \\
\hline Producción & 3 & 4 & 3 & 4 & 14 \\
\hline Revisión & 12 & 7 & 2 & 5 & 26 \\
\hline Social12 & 2 & 2 & 0 & 0 & 4 \\
\hline Total & 24 & 14 & 5 & 12 & 55 \\
\hline
\end{tabular}

Fuente: elaboración propia

En cuanto al subproceso de revisión, se ha indicado que comprende dos acciones: evaluar y corregir. En el análisis del historial del grupo seleccionado hemos advertido únicamente la acción de corregir, sin embargo, suponemos que dichas correcciones han pasado previamente por una evaluación. Es trascendental destacar que tales evaluaciones se han hecho individualmente y que los estudiantes no han negociado los contenidos con sus compañeros. Es debido a la ausencia de una regulación interactiva entre los participantes que ha existido una escasa autorregulación.

Efectivamente, los registros del historial no muestran comentarios que manifiesten dudas o inquietudes respecto a lo que han escrito antes sus pares; incluso, los participantes se centran directamente en la corrección. Tal como indica Arenas (2017), desde la regulación socialmente compartida las instancias de negociación acerca de las acciones por realizar son fundamentales. Sin embargo, en el wiki del Equipo 1, tal como se aprecia en la captura de pantalla de las modificaciones realizadas por uno de los participantes durante la segunda fase de escritura (ver Figura 2), el participante que está editando no indica comentario o sugerencias al aporte realizado por su compañero previamente, sino que modifica el contenido sin negociación. En color rojo se marca lo que el estudiante ha borrado y en color verde lo que ha agregado. De este modo, el texto final queda de la manera que se muestra en la Figura 3.

\footnotetext{
${ }^{12}$ Entendemos por comentario social todo aquel que incluye saludos, bromas o elementos que no tengan relación directa con el desarrollo de la actividad.
} 


\title{
Fuente: Historial del wiki del Equipo 1
}

un motivo por el cual se realiza, la comida esta presente y un muchos de los casos, suele superar en importancia al espíritu de la festividad.

realizar en la sociedad y especialmente-ndesde el mismo estado, dando un enfoque especial a los niños.(fuente:https://scielo.conicyt.el/scielo.php?seript=sci_arttext\&pid=\$0717 $75182002000100008)$. Con esto tambiénniños, otorgándoles el alimento necesario, como se hizo durante el gobierno de Allende donde todos los niños tenían el derecho a medio litro de leche diario.Llevando este punto a nuestros días, se da paso a un sobre alimentación, especialmente-en tos niños, pere al noseguir creyendo que ser "rellenito" es por tenertos recursos necesarios pero esto es solamente el reflejo de una sociedad altamente desnutrida y con malos hábitos alimenticios que buscaba aumentarbuena salud. Pero la ealidad de vida de sus hijos at sobrealimentarlos, yalimentación actual se basa en comida no sólocontentos con eso, hacerlos de mala manera al incrementar susaludable, incrementando la ingesta de grasa,ealoriasgrasa, calorías y azúcares. Todeazúcares. Todo esto se ve reflejado corazón contento" y además, que se generen idolos enídolosen la figura de la

En tercer lugar, la comida también se ve como un eje central en la religión, partiendo de la misma última cena, donde Jesús y sus discípulos comieron por última vez todos juntos. Este hecho para

Figura 2. Captura de pantalla de las modificaciones realizadas por uno de los participantes en el wiki sin negociación

\section{Fuente: Historial del wiki del Equipo 1}

\begin{abstract}
En un segundo lugar, culturalmente en Chile se creía y se cree que ser "gordito" o "rellenito" es un síntoma de buena salud. Esto se debe a que en el siglo pasado, gran parte de la población no tenía con que alimentarse por lo que la tasa de desnutrición era muy alta, cosa que fue cambiando con el paso de los años, gracias a la intervención que se tuvo que realizar en la sociedad desde el mismo estado, dando un enfoque especial a los niños, al otorgándoles el alimento necesario, como se hizo durante el gobierno de Allende donde todos los niños tenían el derecho a medio litro de leche diario. Llevando este punto a nuestros días, se da paso a un sobre alimentación, al seguir creyendo que ser "rellenito" es por tener una buena salud. Pero, la alimentación actual se basa en comida no saludable, incrementando la ingesta de grasa, calorías y azúcares. Todo esto se ve reflejado en que se genere la frase "güatita llena, corazón contento", y que además, se generen ídolos en la figura de la comida rápida, ya que son aspiraciones de la clase baja a parecerese a la clase alta, debido a que en un principio, solo estas últimas tenían la capacidad de comprar una cajita feliz, una Whopper o una BigMac debido a su alto precio de adquisición.
\end{abstract}

Figura 3. Captura de pantalla de fragmento del texto final del wiki

Por otra parte, al igual que en el análisis de los subprocesos realizados, la revisión que hizo cada participante pudo constar de la corrección de más de un nivel textual. Es decir, un participante pudo modificar en una de sus intervenciones tanto el nivel de oración como errores ortográficos y signos de puntuación (ver Figura 4). 
Fuente: Historial del wiki del Equipo 1

\section{Comparing versions of FrontPage}

Showing changes between June 29, 2018 at 5:52:35 pm (erossed out) and June 29, 2018 at 5:56:35 pm (underlined)

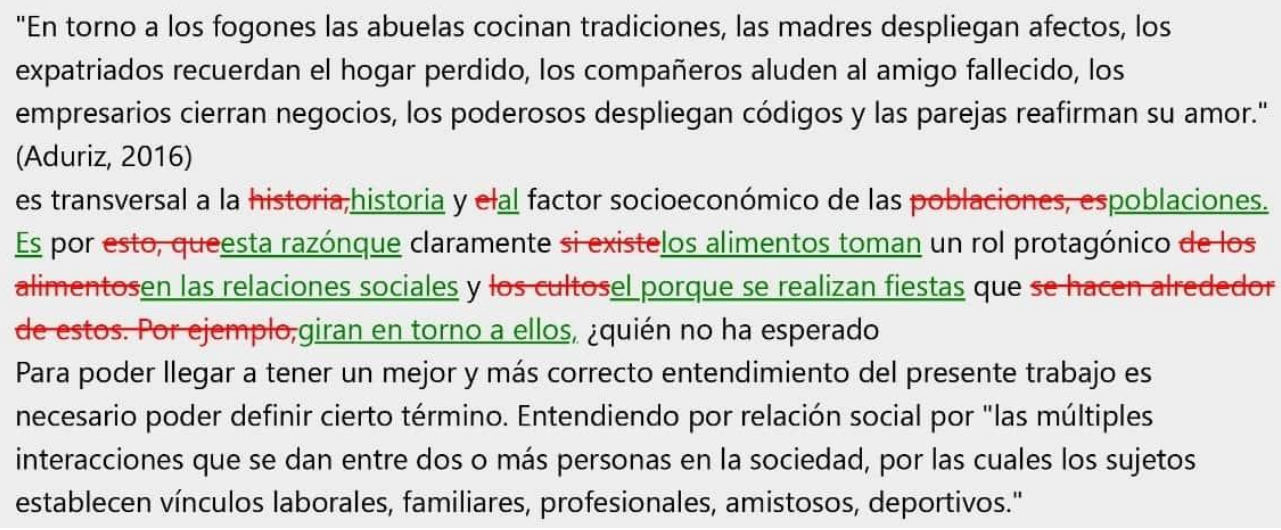

Figura 4. Captura de pantalla de las modificaciones realizadas por uno de los participantes en el wiki en diferentes niveles textuales

En relación con esta idea, aunque las revisiones pudieran pertenecer a más de un nivel textual, luego hacer un análisis de contenido de cada uno de los cambios realizados y comparar todas las versiones de la construcción del texto, observamos a través de la función de comparación del historial del wiki que la mayoría de las correcciones corresponden a la forma más que al contenido. Aunque los dos participantes que están más implicados en la tarea hacen en total cuatro reformulaciones del contenido, las demás variaciones corresponden únicamente a modificación de conectores, de palabras o de ortografía (ver Tabla 3). Estas cifras dan cuenta de que una vez que los estudiantes han planificado el contenido del ensayo sí vuelven muchas veces al texto: lo releen y modifican ciertos aspectos superficiales, aunque esta relectura no implica una modificación de las estructuras cognitivas.

Tabla 3. Revisiones de los participantes del Equipo 1 según el nivel textual de intervención

\begin{tabular}{l|lllll}
\hline Nivel de revisión & M.G. & M.X. & M.O. & F.E. & Total \\
\hline Contenido & 3 & 1 & 0 & 0 & 4 \\
Párrafo & 0 & 1 & 0 & 0 & 1 \\
Oración & 5 & 3 & 1 & 4 & 13 \\
Palabra & 2 & 4 & 0 & 2 & 8 \\
Sílaba/letras & 3 & 1 & 2 & 1 & 7 \\
Paratextos & 2 & 0 & 0 & 1 & 3 \\
Total & 15 & 10 & 3 & 8 & 36 \\
\hline
\end{tabular}

Fuente: elaboración propia 


\section{DisCUSIÓN DE LOS RESULTADOS}

En vista de los datos obtenidos, las revisiones de los estudiantes obedecen primordialmente a correcciones superficiales, pues no tienen una evaluación formadora que los guíe y oriente en el proceso de enseñanza-aprendizaje. Las indicaciones de la maestra señalan recomendaciones poco claras y no hay pautas concretas que enseñen a los estudiantes los códigos de revisión que deben aplicar en el proceso: no hubo comentarios sobre las fuentes consultadas y tampoco sugerencias con respecto a la tesis planteada por cada equipo. En otras palabras, se requiere más que una constatación de avances para que los participantes puedan apreciar en la tarea de escritura un proyecto de aprendizaje y de proceso y no solo un producto que será calificado. En este aspecto, consideramos esencial que el docente oriente al aprendiz en su proyecto de escritura a través de un modelado o modelaje (López et al., 2019), con el objetivo de entregar al escritor novel sucesivas respuestas a lo largo de todo el proceso, así como también propuestas que lo ayuden a evaluar y corregir sus textos.

Igualmente, la utilización del instrumento de evaluación no ha sido adecuada, puesto que se presenta solo al final del proyecto. Según Ribas (1997), en el aprendizaje de la escritura el material de evaluación debería dar ciertas respuestas a los estudiantes y al profesor. Para los primeros, tendría que explicitar las características del texto; la valoración de estos elementos por medio de la negociación de criterios de evaluación; y entregar propuestas de cambio y mejora del proceso (propuestas de corrección). Para los docentes, la evaluación debe permitir conocer el proceso de aprendizaje de los alumnos para determinar de qué modo puede ayudarlos a mejorar.

En este sentido, cabe aludir a la concepción de evaluación de Gil (2019), quien la considera un proceso integral que permite obtener y procesar información para mejorar tanto la enseñanza como el aprendizaje. Se desprende de los datos obtenidos y del material de evaluación dado por la profesora (pauta de cotejo) que no basta la verificación de presencia o ausencia de elementos, a menos que los estudiantes lo empleen durante su proceso de escritura, junto con la aportación de evidencias de los indicadores. A su vez, estos indicadores deben ser, como hemos observado antes, negociados previamente con los discentes. Sin lugar a duda, es mucho más beneficioso para el estudiantado conocer y utilizar el instrumento de evaluación antes, durante y después del proceso de escritura.

Uno de los elementos que, pese a no haber sido objeto de estudio, sí ha tenido una influencia determinante es la utilización del género ensayo. Como género académico se utiliza en todo el currículum escolar, especialmente en las áreas de carácter humanista. Al respecto, la profesora indicó que los estudiantes son regularmente sometidos a la escritura de este tipo de textos, aun cuando previamente no se les había enseñado la estructura: "Ellos han hecho muchos ensayos, pero nunca les han enseñado la estructura" (fragmento de entrevista a la docente).

A partir de lo anterior, debemos tomar consciencia de que el género condicionará el proceso de escritura y los mecanismos de revisión que se apliquen. Es necesario, en consecuencia, hacer un análisis de la naturaleza sociodiscursiva del texto 
que se está produciendo, puesto que, como indica Allal (2018), el género del texto es un vehículo de mediación en una lengua. En otras palabras, se vuelve fundamental que los alumnos también tengan esta consideración y empleen estrategias o códigos de revisión apropiados al género del discurso o a la tarea de escritura concreta a la que se enfrentan. Sin embargo, la profesora indicó que los estudiantes presentan grandes dificultades para este subproceso, ya que no están seguros de sus acciones y, en alguna medida, la revisión es un subproceso que solo el maestro estaría capacitado para llevar a cabo.

Por su lado, los participantes del "Equipo 1" afirmaron que su proceso de escritura se vio mayormente determinado por tres subprocesos: el acceso al conocimiento, la producción y la revisión. En cuanto al subproceso de revisión, hay una disonancia con la percepción que la profesora posee, pues los jóvenes afirman que lo importante sería sobre todo el contenido de los textos y la comprensión de estos por parte de los lectores. No obstante, pese a ello, no se observa que posean orientaciones concretas acerca de cómo alcanzar esta finalidad. En este sentido, se vuelve fundamental conocer cuáles son los mecanismos de evaluación que se llevan a cabo en el subproceso de revisión y también de qué manera se enseña a los alumnos a auto- y corregular la producción de sus textos.

Ciertamente, desde la perspectiva sociocultural y de la regulación socialmente compartida, la escritura es una práctica dialógica y situada, que requiere mediación. Por lo tanto, la regulación externa de las prácticas de escritura colaborativa debería caracterizarse por el establecimiento de objetivos comunes por parte de los miembros del equipo y por su interacción simétrica con la finalidad última de integrar los conocimientos propios con los de los demás integrantes.

En nuestros datos el trabajo colaborativo presenta una gran problemática: no parece que los estudiantes desarrollen corregulación ni autorregulación. Aparentemente, no discuten con sus compañeros ideas ni contenidos en la construcción del texto. De este modo, el proceso de producción conjunto se limita a la mera división de partes de un trabajo que luego se unen de manera sucesiva. En este sentido, la corregulación, esto es, el fomento u obstaculización de la regulación contextual se ve limitada y logra poca influencia en la regulación interna o autorregulación. Los estudiantes participantes del estudio toman poca conciencia del conocimiento lingüístico y relativo al aprendizaje (Allal, 2018, Arenas, 2017, López et al., 2019).

Para terminar, la profesora indicó que los estudiantes presentaron muchas dificultades para el manejo del wiki, puesto que sus conocimientos respecto a herramientas de la Web 2.0 estarían predominantemente inclinados tan solo al uso de redes sociales. Además, trabajar un texto digitalmente cambiaría su proceso de producción en tanto verían la construcción de los avances como textos finales y no como borradores susceptibles de mejoras y cambios. En relación con el dominio de esta herramienta, los estudiantes coincidieron con la docente en que tuvieron dificultades. No obstante, admiten que producir textos en formatos digitales es más fácil que en soporte papel: "es más ordenado" (fragmento de entrevista a estudiante); "Yo creo que 
es más fácil porque uno puede trabajar sin perder lo anterior o borrar, puedo corregir sobre lo que ya hice" (fragmento de entrevista a estudiante). Asimismo, la utilización de elementos multimodales (imágenes, enlaces, vídeos, etc.) favorecería el acceso al conocimiento.

A partir de este estudio se desprende que la escritura digital favorece la multidimensionalidad de recursos. Sin embargo, se requieren más investigaciones y apoyo para capacitar tanto a los discentes como a los docentes en la integración eficiente y eficaz de los recursos digitales a las aulas. En este sentido, nos referimos a que la inmersión de la Web 2.0 en las aulas requiere cumplir ciertas condiciones que permitan potenciar en el estudiante una visión de esta como lo que es: una herramienta al servicio de su aprendizaje.

De esta circunstancia se deduce que todavía sea necesaria una inversión en la capacitación de docentes para que dichos recursos digitales sean utilizados provechosamente. Adecuados son los aspectos que Moreno (2012) señala que se debe tener presentes si se quiere instaurar la Web 2.0 dentro del proceso de enseñanzaaprendizaje: modificación de rol del educador y del educando; pasar de la enseñanza tradicional al creador de conocimientos e investigador; un cambio de metodología y nuevos estilos de aprendizaje; formación docente; y el desarrollo de nuevas competencias.

Finalmente, trabajar en un soporte digital con la posibilidad de que los textos sean posteriormente publicados otorga un carácter motivacional a la tarea de escritura. Los estudiantes señalan que cuando realizan trabajos en los que invierten mucho esfuerzo quisieran que estos sean conocidos y comentados por otros. Se observa que es fundamental otorgar espacios de escritura que tengan como destinatario final no solo al profesor que calificará su trabajo, sino que se ofrezcan escenarios reales de interacción que instarán a los jóvenes a escribir con mayor ahínco y con la motivación de compartir y colaborar en la producción de conocimiento.

\section{CONCLUSIONES}

Hasta ahora hemos, en primer lugar, indagado sobre las percepciones de los participantes en el estudio acerca del proceso de escritura colaborativa, en su participación en redes sociales y en su uso de sitios Web 2.0. Se concluye que los estudiantes aprecian el trabajo colaborativo como un modo que les permite aprender más. En concreto, son muchos los beneficios a nivel cognitivo porque invita a la reflexión y discusión entre los integrantes del grupo. Sin embargo, al menos a nivel de escritura, según los cambios registrados a través del historial del wiki, los estudiantes no realizan un trabajo solidario entre las partes. Por consiguiente, se debe generar un traspaso progresivo de la responsabilidad del docente al alumno, para que sea este último quien pueda corregular a sus compañeros hasta finalmente incorporar el proceso y poder autorregularse.

Por otro lado, los estudiantes que forman parte de la muestra informan participar y usar las herramientas de la Web 2.0, principalmente a través de las redes 
sociales. Pese a ello, no existiría un alto dominio de otros soportes multimodales. Con todo, reconocen que espacios como el wiki ofrecen elementos multimodales que facilitarían sus procesos de escritura, pues el soporte cambiaría los procesos de revisión que se ejecutan en el formato papel y son espacios que representarían una motivación intrínseca para escribir, porque la publicación de los textos como producto social los animaría como escritores en formación a impactar en un espacio real y tener una retroalimentación de sujetos que no solo buscan calificar su producto final.

En segundo lugar, se concluye que los estudiantes son conscientes de que existe un subproceso de revisión y lo llevan a cabo mediante la evaluación y corrección de elementos que consideran erróneos. No obstante, los elementos en los que centran su atención son principalmente de forma, pues, aunque señalan que lo importante es el contenido, no tienen claros qué códigos de revisión deben aplicar, ni conocen estrategias que les ayuden a materializar los conceptos abstractos de coherencia y cohesión al texto real que están construyendo. Además, tampoco consideran dentro del proceso de construcción la posibilidad de volver a otros procesos previos, como la planificación o el acceso al conocimiento. Más bien, siguen etapas sucesivas y cuando tienen un primer borrador, solo evalúan y corrigen a nivel superficial. De hecho, no muestran cambios de párrafos, supresión o adición de contenido. En el mejor de los casos hay reformulación de lo que habían escrito al inicio del proceso.

Debido a que este estudio solo representa una aproximación al subproceso de revisión en la escritura colaborativa digital, quedan muchas cuestiones por resolver. Consideramos que será interesante investigar cómo se puede enseñar a los estudiantes la aplicación de códigos de revisión colaborativa (modelado o modelaje). Las investigaciones en cuanto al subproceso de revisión deben centrarse no solo en qué, cómo o para qué revisar, sino que también se deben ampliar e investigar el papel que ejerce el profesor como mediador en este proceso de enseñanza de los códigos de revisión para con el alumno.

Asimismo, será necesario generar metodologías que promuevan el trabajo en equipos consolidados que puedan emprender un proyecto con responsabilidad y solidaridad, más que como una manera de organización, como una competencia para la vida. Finalmente, el dominio de las herramientas digitales nos lleva a la necesidad de estudiar la disposición y usos de los jóvenes frente a la utilización de las herramientas Web 2.0 en tareas pedagógicas.

\section{REFERENCIAS}

AERA Council. (2011). Code of Ethics American Educational Research Association. Educational Researcher, 40(3), 145-156. doi: 10.3102/0013189X11410403.

Allal, L. (2018). The co-regulation of writing activities in the classroom. Journal of Writinng Research, 10(1), 25-60. doi: 10.17239/jowr-2018.10.01.02.

Álvarez, T. (2010). Reescribir para escribir textos en las aulas. Textos, (53), 102-118. 
Álvarez, T., y González, M. (2012). RedacText, guía on line para ayudar a redactar. RELADA, 6(2), 167-174. Recuperado de http://polired.upm.es/index.php/relada/article/view/1900.

Arenas, K. (2017). Características de la regulación socialmente compartida de la escritura: aportes desde una experiencia investigativa. Lenguaje, 45(1), 35-60. doi: 10.25100/lenguaje.v45i1.4613.

Cabrera, E. (2004). Aprendizaje colaborativo soportado por computador (CSCL): su estado actual. Revista Iberoamericana de Educación, 34(1), 1-16. doi: 10.35362/rie3412933.

Caldera, R., y Escalante, D. (2006). Escribir en el aula de clase: Diagnóstico en sexto grado de Educación Básica. Revista de Pedagogía, 27(80), 371-405. Recuperado de https://www.redalyc.org/articulo.oa?id=65908002.

Calle, G. (2018). La escritura académica apoyada por un Centro de Escritura Digital en la educación media. Lenguaje, 46(2), 334-361. doi: 10.25100/lenguaje.v46i2.6586.

Cassany, D., y López, C. (2005). Tareas para la producción de escritos en un Centro de Redacción virtual. En G. Vázquez (Coord.), Español con fines académicos: de la comprensión a la producción de textos (pp. 153-176). Madrid: Edinumen.

Castelló, M., Bañales, G., y Vega, N. (2010). Research approaches to the regulation of academic writing: the state of the question. Electronic Journal of Research in Educational Psychology, 8(22), 1253-1282. doi: 10.25115/ejrep.v8i22.1424.

Cohen, L., Manion, L., y Morrison, K. (2011). Research Methods in Education (7a ed.). Londres: Routledge.

Collazos, C., y Mendoza, J. (2006). Cómo aprovechar el "aprendizaje colaborativo" en el aula. Educación y Educadores, 9(2), 61-76. Recuperado de https://educacionyeducadores.unisabana.edu.co/index.php/eye/article/view/663

Creswell, J. W. (2007). Qualitative Inquiry Research Design. Choosing Among Five Approaches ( $2^{\mathrm{a}}$ Ed.). California: SAGE Publications.

Creswell, J. W. (2012). Educational research: Planning, conducting, and evaluating quantitative and qualitative research ( $4^{\mathrm{a}} \mathrm{ed}$.). Boston: Pearson.

De Caso, A., y García, J. (2006). Evolución de los modelos de escritura. International Journal of Developmental and Educational Psychology, 2(1), 523-532. Recuperado de https://www.redalyc.org/pdf/3498/349832312044.pdf.

Dörnyei, Z. (2007). Research Methods in Applied Linguistics. Quantitative, Qualitative, and Mixed Methodologies. Oxford: Oxford University Press.

Duffy, P., y Bruns, A. (2006). The Use of Blogs, Wikis and RSS in Education: A Conversation of Possibilities. En A. Brown (Ed.), Learning on the Move: Proceedings of the Online Learning and Teaching Conference 2006 (pp. 31-38). Brisbane, Australia.

Flower, L. (1979). Writer-Based Prose: A Cognitive Basis for Problems in Writing. College English, 41(1), 19-37. doi: 10.2307/376357.

Flower, L., y Hayes, J. R. (1981). A Cognitive Process Theory of Writing. College Composition and Communication, 32(4), 365-387. doi: 10.2307/356600.

García, B. C., López, M. C., y García, A. (2014). Los riesgos de los adolescentes en 
Internet: los menores como actores y víctimas de los peligros de Internet. Revista Latina de Comunicación Social, (69), 462-485. doi: 10.4185/RLCS-2014-1020.

Gil, J. (2019). Representaciones sociales de la evaluación en dos Escuelas de Secundaria de Guadalajara, México. Lenguaje, 47(1), 173-200. doi: 10.25100/lenguaje.v47i1.7321.

Grupo Didactext. (2003). Modelo sociocognitivo, pragmalingüístico y didáctico para la producción de textos escritos. Didáctica. Lengua y Literatura, 15, 77-104. Recuperado de https://revistas.ucm.es/index.php/DIDA/article/view/20310.

Guitert, M., y Pérez-Mateo, M. (2013). La colaboración en la red: Hacia una definición de aprendizaje colaborativo en entornos virtuales. TESI, 14(1), 10-31. doi: 10.14201/eks.9440.

Guzmán, K., y Rojas-Drummond, S. (2012). Escritura colaborativa en alumnos de primaria. Revista Mexicana de Investigación Educativa, 17(52), 217-245. Recuperado de http://www.scielo.org.mx/scielo.php?script=sci_arttext\&pid=S140566662012000100010.

Johnson, D. W., Johnson, R. T., y Smith, K. A. (1991). Cooperative Learning: Increasing College Faculty Instructional Productivity. ASHE-ERIC Higher Education Report no 4. Washington D. C.: The George Washington University.

Latorre, M. (2018). Historias de las Web, 1.0, 2.0, 3.0, 4.0. Recuperado de http://umch.edu.pe/arch/hnomarino/74_Historia\%20de\%20la\%20Web.pdf.

López, C., Martín, E., Esteve, O., y Atienza E. (2019). La competencia discursiva en sus constelaciones: glosario. Recuperado de https://www.upf.edu/web/ecodal/glosario.

Lucero, M. (2003). Entre el trabajo colaborativo y el aprendizaje colaborativo. Revista Iberoamericana de Educación, 33(1), 1-21. doi: 10.35362/rie3312923.

McDonough, K., De Vleeschauwer, J., y Crawford, W. (2018). Comparing the quality of collaborative writing, collaborative prewriting, and individual texts in a Thai EFL context. System, 74, 109-120. doi: 10.1016/j.system.2018.02.010.

Ministerio de Educación. (2015). Bases Curriculares $7^{\circ}$ básico a $2^{\circ}$ medio. Santiago de Chile: Unidad de Currículum y Evaluación.

Moreno, A. (2012). La web 2.0. Recurso educativo. Recuperado de: http://recursostic.educacion.es/observatorio/web/es/internet/web-20/1060-laweb-20-recursos-educativos.

O'Reilly, T. (2007). What Is Web 2.0: Design Patterns and Business Models for the Next Generation of Software. International Journal of Digital Economics, (65), 17-37. Recuperado de https://mpra.ub.uni-muenchen.de/id/eprint/4578.

Ortiz, M., y Ferreira, A. (2014). Proposing a wiki-based technique for collaborative essay writing. Profile: Issues in Teachers' Professional Development, 16(2), 185-198. doi: 10.15446/profile.v16n2.38877.

Panadero, E., Järvelä, S., Malmberg, J., Koivuniemi, M., Phielix, C., Jaspers, J., y Kirschner, P. (2013). Enhancing socially shared regulation in working groups using a CSCL regulation tools. En E. Walker y C.-K. Looi (Eds.), Proceedings of the Workshops at the 16th International Conference on Artificial Intelligence in Education AIED (pp. 7-12). Memphis, USA. 
Peachey, N. (2009). Web 2.0 Tools for Teachers. Recuperado de: https://es.scribd.com/doc/19576895/Web-2-0-Tools-for-Teachers.

Pujolà, J.-T. (2013). Autonomía y uso de las TIC. En O. Esteve y E. Martín-Peris, Cuestiones de Autonomía en el Aula de Lenguas Extranjeras (pp. 67-85). Barcelona: Horsori.

Ribas, T. (1997). Evaluar en la clase de lengua: cómo el alumno gestiona su proceso en la escritura. Textos, (11), 53-65.

Roselli, N. (2011). Teoría del aprendizaje colaborativo y teoría de la representación social: convergencias y posibles articulaciones. Revista Colombiana de Ciencias Sociales, 2(2), 173-191. Recuperado de https://www.funlam.edu.co/revistas/index.php/RCCS/article/view/238.

Scardamalia, M., y Bereiter, C. (1992). Dos modelos explicativos de los procesos de composición escrita. Journal for the Study of Education and Development, 15(58), 4364. doi: 10.1080/02103702.1992.10822332.

Storch, N. (2013). Collaborative Writing in L2 Classrooms. Bristol: Multilingual Matters.

Storch, N. (2019a). Collaborative writing. Language Teaching, 52(1), 40-59. doi: $10.1017 /$ S0261444818000320.

Storch, N. (2019b). Collaborative Writing as Peer Feedback. En K. Hyland y F. Hyland (Eds.), Feedback in Second Language Writing. Context and Issues (2a ed.) (pp.143-162). Nueva York: Cambridge University Press.

Subsecretaría de Telecomunicaciones de Chile. (2017). IX Encuesta de Acceso y Usos de Internet. Recuperado de https://www.subtel.gob.cl/wpcontent/uploads/2018/07/Informe_Final_IX_Encuesta_Acceso_y_Usos_Internet_ 2017.pdf.

Tapia, M., Correa, R., Ortiz, M., y Neira, A. (2012). Secuencia didáctica para escritura en colaboración en un entorno wiki: Propuesta para estudiantes de Enseñanza Secundaria. Revista Española de Pedagogía, 70(253), 337-353. Recuperado de https://revistadepedagogia.org/lxx/no-253/secuencia-didactica-para-escrituraen-colaboracion-en-un-entorno-wiki-propuesta-para-estudiantes-de-ensenanzasecundaria/101400010266/.

Trujillo, F. (2002). Aprendizaje cooperativo para la enseñanza de la lengua. Publicaciones, 32, 147-162. Recuperado de https://revistaseug.ugr.es/index.php/publicaciones/article/view/2329.

Ubilla, L., Gómez, L., y Sáez, K. (2017). Escritura colaborativa de textos argumentativos en inglés usando Google Drive. Estudios Pedagógicos, 43(1), 331-348. doi: 10.4067/S0718-07052017000100019.

Uribe, A., Ramírez, D., y Henao, O. (2017). Exploración de un ejercicio de escritura colaborativa en línea de un grupo de estudiantes de básica primaria. Revista Lasallista de Investigación, 14(1), 29-41. doi: 10.22507/rli.v14n1a2.

White, D. S., y Le Cornu, A. (2011). Visitors and Residents: A new typology for online engagement. First Monday, 16(9). doi: 10.5210/fm.v16i9.3171.

Zañartu, L. (2003). Aprendizaje colaborativo: una nueva forma de Diálogo Interpersonal $\mathrm{y}$ en Red. Contexto Educativo, 5(28). Recuperado de 
http://files.enriquecer-educaciontic.webnode.es/200000026-

9a1009c069/Contexto_Educativo_-

_Revista_digital_de_Educacion_y_Nuevas_Tecnologias.pdf.

\section{SOBRE LA AUTORA}

\section{Emmy Antonella González Lillo}

Profesora de Castellano y Comunicación, Licenciada en Educación por la Pontificia Universidad Católica de Valparaíso y Máster en Investigación en Didáctica de la Lengua y la Literatura por la Universidad de Barcelona. Actualmente cursa el Programa de Doctorado en Traducción y Ciencias del Lenguaje de la Universitat Pompeu Fabra financiada por el sistema Becas-Chile de la Agencia Nacional de Investigación y Desarrollo de Chile. Áreas de interés académico: enseñanza y evaluación de la escritura y escritura colaborativa.

Correo electrónico: emmy.gonzalez@upf.edu

Orcid: 0000-0001-8934-1968. 